\title{
Tsafon
}

Revue d'études juives du Nord

$74 \mid 2017$

La Déclaration Balfour, vers l'État d'Israël ?

\section{Theodor Lessing. Lettre inédite de Jérusalem}

\section{Martine Benoit}

\section{OpenEdition}

Journals

Édition électronique

URL : https://journals.openedition.org/tsafon/411

DOI : 10.4000/tsafon.411

ISSN : 2609-6420

\section{Éditeur}

Association Jean-Marie Delmaire

\section{Édition imprimée}

Date de publication : 1 décembre 2017

Pagination : 143-150

ISSN : 1149-6630

\section{Référence électronique}

Martine Benoit, «Theodor Lessing. Lettre inédite de Jérusalem», Tsafon [En ligne], 74 | 2017, mis en ligne le 31 mai 2018, consulté le 01 juillet 2021. URL : http://journals.openedition.org/tsafon/411 DOI : https://doi.org/10.4000/tsafon.411 


\section{Édition : Theodor Lessing}

\section{Theodor Lessing Lettre inédite de Jérusalem}

Martine Benoit*

Intellectuel incarnant la figure judéo-allemande et son échec, Theodor Lessing (1872-1933) est un enfant mal aimé de l'histoire. La postérité n'a voulu retenir de la vie de cet homme, journaliste, essayiste et professeur de philosophie, que deux événements : le titre accrocheur de son dernier ouvrage, La haine de soi juive; l'assassinat par des hommes de main des nazis le 30 août 1933 sur le sol tchécoslovaque, à Marienbad. Le document que nous traduisons et publions ici fait référence à un épisode moins connu de la vie de Theodor Lessing : son voyage en Palestine.

Au printemps 1931, Theodor Lessing s'éloigne de l'Université de Hanovre où il enseigne depuis 1907 ; des étudiants ultra-nationalistes menacent en effet d'attenter à sa vie. Il entame un long voyage qui lui fait découvrir l'Égypte, la Palestine, la Grèce et Salonique. Vers le 25 mars 1931, il embarque à Trieste, débarque le mercredi $1^{\text {er }}$ avril à Alexandrie et continue vers Le Caire. Le 4 avril, il part pour El-Kantara afin, une fois

\footnotetext{
* Université de Lille, directrice de la Maison Européenne des Sciences de l'Homme et de la Société.
} 
le Nil traversé, de prendre le train pour la Palestine. Il arrive le dimanche de Pâques à Jérusalem où il reste jusqu'au 10 avril. Le vendredi 10, il pénètre dans Tel-Aviv, silencieuse et solennelle en ce soir de shabbat. Du mardi 14 au mardi 21 avril, il séjourne à Haïfa pour s'installer finalement pendant quatre jours sur les bords du lac de Tibériade. Le 24 avril, il part pour Damas puis Beyrouth, la troisième étape de son voyage devant le mener, après Chypre, Rhodes et Le Pirée (voyage en bateau du $1^{\mathrm{er}}$ au 10 mai), à Salonique où sa fille ainée, Judith, vit mariée à un Grec. Le moment du retour approche cependant; le 13 juin, Theodor Lessing embarque pour Istanbul où, après un séjour de cinq jours, il prend le train pour Sofia, puis Budapest, Berlin et Hanovre ${ }^{1}$.

Pendant son périple, Theodor Lessing adresse de nombreuses lettres à sa femme Ada et à sa fille Ruth. Il demande d'ailleurs expressément à ces dernières de conserver soigneusement ces feuillets dont il souhaite «[se] servir plus tard comme points de repère pour raconter et même peut-être pour mettre par écrit [ses] impressions $»^{2}$. Les lettres, signées alternativement « le vieux loup de mer », « le fidèle loup de mer» ou «Theodorus Jerusalemitius », sont développées, pleines d'anecdotes et d'observations.

Le document présenté ici est consacré à l'arrivée de Theodor Lessing à Jérusalem et au début de son séjour. Cette première lettre écrite de Palestine est un bon condensé des missives que Theodor Lessing envoya durant son voyage : il se montre inhabituellement enjoué ; il apprécie le climat chaud; il rencontre de nombreuses personnes et aime être reconnu et salué; c'est en touriste qu'il visite la Palestine. Le document original a été traduit de l'allemand. Cette traduction prélude à la publication de l'ensemble de ces lettres.

\footnotetext{
${ }^{1}$ Trajet reconstitué, non sans peine (Theodor Lessing donne peu de détails sur ses trajets, il se trompe dans les dates), à partir des lettres de Theodor Lessing à sa famille. ${ }^{2}$ Lettre adressée à Ada et Ruth Lessing de Jérusalem le 7 avril 1931, page numérotée 8. Pour aider dans son travail un père qu'elle avait l'habitude d'accompagner et de seconder dans ses voyages en Europe, Ruth Lessing s'est empressée de porter au propre, en les tapant à la machine, ces longues missives.
} 
Jérusalem, mardi le 7 avril $1932^{3}$

Dès les premiers pas, je me sentis accueilli et en sécurité. On sentait tout de suite qu'on était en pays juif ${ }^{4}$. Tout était écrit en langue arabe, hébraïque et anglaise mais l'élément hébraïque est sans aucun doute dominant et bien plus intelligent. Il est vrai que chaque Juif comprend un peu d'allemand. Je me rendis à l'un des hôtels conseillés ${ }^{5}$ où je m'installai dans une chambre propre et peu coûteuse. Je me lavai et me changeai. On était dimanche de Pâques et il y avait une cérémonie dans l'église du Saint-Sépulcre au pied de la tombe de Jésus. Mais j'étais si fatigué que je m'endormis immédiatement. À mon réveil, il était déjà midi. Après le déjeuner, je me rendis immédiatement au bureau d'information sioniste. J'y fus reçu par un certain monsieur Flessner (ou quelque chose comme ça), un ami proche de Werner Kraft. Puis je partis

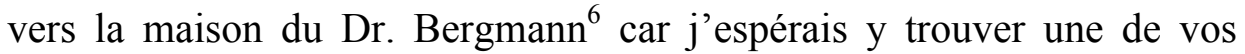
lettres. Il m'accueillit et m'invita à passer la journée avec lui. Nous prîmes la voiture pour aller au Mont Scopus et nous visitâmes d'abord l'université et la bibliothèque à l'aménagement tout moderne ${ }^{7}$ et la collection des œuvres complètes de tous les auteurs juifs de la terre ; d'immenses masses de livres. Bergmann me montra aussi l'emplacement de mes livres. Mes livres sur la philosophie de l'histoire étaient près de ceux de Scheer $^{8}$, ce qui me fit très plaisir. Mais je vis dans les catalogues

\footnotetext{
${ }^{3}$ Cette traduction est publiée avec l'amicale autorisation du Dr. Christian Heppner, responsable des Archives municipales de la Ville de Hanovre (Stadtarchiv Hannover), que nous remercions ici particulièrement. Cette lettre est conservée au Stadtarchiv Hannover sous la cote : 3 NL 125 Lessing Nr. 1050.

${ }^{4}$ Theodor Lessing vient d'Égypte et donc souligne ici ce qu'il ressent comme un contraste.

${ }^{5}$ Avant de prendre le train à El-Kantara, Theodor Lessing est entré, dans la gare, dans un bureau de l'Agence juive où il a pu prendre un guide de la Palestine juive avec des renseignements sur les hôtels juifs de Jérusalem.

${ }^{6}$ Le docteur Bergmann était un ami de la famille.

${ }^{7}$ L'Université hébraïque de Jérusalem venait d'ouvrir. Située sur le mont Scopus dominant la vieille ville, elle fut inaugurée en 1925.

${ }^{8}$ Johannes Scherr (1817-1886), révolutionnaire déçu de 1848, a été une sorte de maître à penser du jeune Theodor Lessing et il eut de plus une influence non négligeable sur sa conception de la philosophie de l'histoire.
} 
qu'à part moi, il y avait encore une série d'autres Lessings qui avaient tous écrit des livres. Je dus ensuite m'inscrire dans le livre d'or de l'université et un hasard bien contrariant fit que mon nom se retrouva en face de celui de Thomas Mann ${ }^{9}$. Puis, du haut du Scopus, nous pûmes voir le coucher de soleil. Devant nous, le Mont des Oliviers, le jardin de Gethsémani, Hiron et la mosquée d'Omar et Jérusalem la ville « élevée »; derrière nous, dans le vide, la montagne de roches grises de la Judée et l'épouvantable Mer morte. Cette université se trouve au point le plus marquant de la planète. Elle est éternelle. Sur le chemin du retour, je fis, grâce à Bergmann, la connaissance de toute sorte de gens. Le fils de Franz Oppenheimer ${ }^{10}$, une homme frais, sain et ouvert, professeur des sciences forestières. Puis dans la rue de Jaffa ${ }^{11}$, nous avons croisé par hasard Dr. Kaznelson ${ }^{12}$, l'éditeur de mon livre juif ${ }^{13}$ que j'avais pu rencontrer à Berlin. Il visite la Palestine avec sa femme. [...]

\footnotetext{
${ }^{9}$ L'inimitié de Theodor Lessing envers Thomas Mann remonte aux années 1900. En 1910, Theodor Lessing écrit dans la revue théâtrale dirigée par Siegfried Jacobsohn, Die Schaubühne, une satire de Samuel Lublinski, alors critique littéraire célèbre. L'exemple de Samuel Lublinski lui sert d'illustration à la figure de l'Espritjude, un concept par lequel il désigne le littérateur juif assimilé, un écrivaillon snob et blasé qui réfléchit peu mais écrit beaucoup, le contre-exemple même du poète véritable. Une bataille littéraire éclate, on parle d'antisémitisme juif, Thomas Mann intervient violemment contre Theodor Lessing qu'il qualifie d'« exemple repoussant de mauvaise race juive ». Pour comprendre la réaction si violente de Thomas Mann, qui commençait alors à être connu, il faut relever que Samuel Lublinski fut l'un des premiers critiques littéraires à accueillir favorablement son roman, Les Buddenbrook ou le déclin d'une famille (1901).

${ }^{10}$ Franz Oppenheimer était un sociologue allemand qui, lors du IX ${ }^{\text {ème }}$ Congrès Sioniste en 1909, proposa de lancer un programme de coopératives agricoles en Palestine (voir Mitchell Cohen, Du rêve sioniste à la réalité israélienne, La Découverte, Paris, 1990).

${ }^{11} \mathrm{La}$ rue de Jaffa à Jérusalem est une rue très animée et prolonge vers l'ouest la sortie de la vieille ville. C'est le long de cette rue que se sont développés les nouveaux quartiers de la ville juive moderne.

${ }^{12}$ Il s'agit de Siegmund Kaznelson, directeur du Jüdischer Verlag entre 1922 et 1924. Le Jüdischer Verlag fut la première maison d'édition sioniste en Europe de l'Ouest, créée par des hommes qui croyaient que la renaissance juive passait par une union entre sionisme politique et sionisme culturel, qu'il fallait donc bâtir un pont entre Westjuden et Ostjuden, entre Juifs occidentaux et Juifs d'Europe orientale. L'idée de ces éditions fut présentée lors du V ${ }^{\text {ème }}$ Congrès sioniste en 1901 et reçut le soutien de Theodor Herzl, Martin Buber faisait alors partie du comité de direction de l'entreprise naissante. Sur le Jüdischer Verlag voir: Anatol Schenker, Der jüdische Verlag 1902-1938. Zwischen Aufbruch, Blüte und Vernichtung, Tübingen, Niemeyer, 2003.

${ }_{13}$ «Mon livre juif» : Theodor Lessing parle ici de son dernier ouvrage, La haine de soi juive, paru à la fin de l'année 1930. La question juive, politiquement stratégique depuis 1929 avec l'effondrement de l'ordre démocratique et la montée de l'extrême-droite, était devenu un sujet débattu dans toute la société allemande. Der jüdische Selbsthass apparait donc comme la contribution de Theodor Lessing au débat sur l'antisémitisme virulent.
} 
Le lendemain, le lundi de Pâques, j'ai pris les services d'un guide. C'était une bonne chose. Sans lui, je n'aurais pu trouver mon chemin dans ce chaos. J'ai assisté à la procession des musulmans vers le tombeau de Moïse, une procession accompagnée de danses et de chants. Mon Dieu ! Les rues étaient pleines de dizaines de milliers de pèlerins. Des types de gens que je n'avais jamais vus. La procession vers la mosquée d'Omar dura des heures car les masses qui dansaient, chantaient et sautaient n'avançaient que très doucement. Des fellahs venus de tous les villages, le drapeau saint, des effendis, des scheiks, tout cela accompagné des troupes anglaises ${ }^{14}$. [...] Puis j'ai visité avec mon guide toute la vieille ville. En commençant par les lieux chrétiens. La Via Dolorosa. Les lieux célèbres du Nouveau Testament. L'église du Saint-Sépulcre. Il faudrait que j'écrive de longues pages là-dessus. Je m'intéresse toujours plus aux gens et à leur manière d'être qu'à l'histoire ou aux légendes des lieux parmi lesquels le plus vieux monument qu'Hélène, la mère de Constantin, fit bâtir, m'apparut vraiment très sain et très achevé. Je vis les plus vieux quartiers juifs et arabes puis pris congé pour quelques heures de mon guide pour aller chez le barbier, aller me laver et manger.

Alors que je faisais une petite promenade après le déjeuner sur la rue de Jaffa, célèbre mais moche (la Georgstrasse de Jérusalem ${ }^{15}$ ), j'entends soudain quelqu'un appeler mon nom par derrière, et quand je me retourne, je vois la famille Kahn, Abrascha, Lilly et leur jeune garçon. Ils avaient lu dans le journal que j'arrivais ce matin et s'étaient empressés de venir de Tel-Aviv et de me chercher. Comme j'avais un guide pour toute la journée, nous décidâmes de passer l'après-midi de manière séparée et de faire le lendemain un tour en voiture. Puis j'allai au Mur des Lamentations. Les gens que j'y vis dépassèrent toutes mes attentes. Cette ferveur dans les prières, ces sanglots, ces pleurs des femmes et des garçons. Et quelles personnalités! Ces sépharades superbes, ces Juives portugaises et sépharades, ces Juifs ashkénazes orthodoxes de Pologne et de Galicie avec leurs pejes ${ }^{16}$ et leurs chapeaux ronds, ces Juifs marocains, sauvages, désolés et affreusement pauvres ; des Juifs persans, européens, des Yéménites, des Syriens, tous unis ici

\footnotetext{
${ }^{14}$ La Palestine était placée sous le régime mandataire de la Grande-Bretagne, c'était donc les forces anglaises qui encadraient ces sortes de manifestations.

${ }^{15}$ Theodor Lessing fait allusion ici à la Georgstrasse de Hanovre qu'il ne semble guère apprécier bien qu'elle fût une des grandes et belles artères de la ville, avec l'opéra et le café Kröpcke, rendez-vous des gens branchés encore aujourd'hui.

${ }^{16}$ C'est-à-dire les papillotes, les mèches de cheveux sur les tempes, typiques des Juifs orthodoxes.
} 
par un destin commun de souffrances. Mais je ne pourrai en parler qu'oralement. Je vis ensuite quelques synagogues, dont une synagogue sépharade. À 18 heures, j'étais dans ma chambre et prenais un bain.

$[\ldots]$

Le lendemain, mardi, j'arrivais à $9 \mathrm{~h}$ à l'hôtel Tel-Aviv pour chercher les Kahn. On était un jour férié, c'était difficile de trouver une voiture. Mais nous finîmes par trouver un vieux fourgon conduit par deux jeunes Juifs effrontés, un Persan et un séfarade. Nous partîmes vers la plaine du Jourdain. [...] Ce désert mort, traître, salé, est monstrueux. C'est là qu'on comprend l'histoire biblique. On comprend que, si elle n'était historique, elle n'aurait pu être inventée autrement. Quand on arrive depuis le désert de Jéricho à Jérusalem, c'est comme si, des profondeurs terribles et de l'enfer, on avançait doucement vers la lumière et la forme. La mer Morte se trouve à plusieurs centaines de mètres endessous du niveau de la mer. Mais entre la mer Morte et Jérusalem on atteint le niveau de la mer. C'est alors que s'élève doucement devant nos yeux Jérusalem, la ville élevée. La mer Morte est épaisse et lourde de sels. C'est en fait une immense réserve souterraine de natron et de sels de potasse, créé par le fleuve Jourdain, capable d'alimenter pour des milliers d'années les hommes en sel et en potasse. Nous avons pu voir les machines avec lesquelles le réservoir de sels de la mer pourra être exploité à l'avenir (une entreprise privée a emporté ce monopole); le procédé lui-même est entouré de mystère ${ }^{17}$. J'ai bu de l'eau de mer brrrr; j'aurais aimé nager ; l'eau est si lourde qu'aucun corps ne peut y couler mais il est très fatigant d'y nager car on doit fendre des masses lourdes. [...] Dans notre fourgon, nous continuâmes sur Jéricho. Ce n'est plus qu'un pauvre village de fellahs aujourd'hui. Avec tout près une petite colonie, Nouveau Jéricho où vivent quelques Anglais et des Allemands. Nous sommes allés jusqu'à la frontière de la Transjordanie. C'est là que commence la région de l'eau, la vallée du Jourdain devient fertile. Nous avons fait une pause près d'une petite source derrière Jéricho. Quelques voitures avec des pèlerins arrivèrent, dont un camion rempli de jeunes prolétaires allemands, un groupe de scouts chrétiens venus en terre sainte pour Pâques ; bien sûr je leur ai demandé si l'un d'eux venait de Hanovre ${ }^{18}$. Mais ils venaient presque tous de Hambourg.

\footnotetext{
${ }^{17}$ Les ressources minérales de la mer Morte seront largement exploitées par l'État d'Israël après l'indépendance du pays.

${ }^{18}$ Theodor Lessing s'est installé en 1907 à Hanovre et y est resté jusqu'au $1^{\text {er }}$ mars 1933 où, poussé par sa fille Ruth, il s'enfuyait à Marienbad.
} 
Le retour, quoiqu'en montée, fut plus court car nous avons rejoint Jérusalem directement. Ce désert autour du plateau de Moab est traître. Il arrive souvent qu'un homme s'y égare - il est alors perdu et meurt vite de soif. Ce sol restera à jamais stérile. Mais on construira des machines et on créera une économie permettant de faire venir l'énergie de loin. Nous mangeâmes à Jérusalem dans le meilleur restaurant de cette rue de Jaffa célèbre mais moche. Puis nous sommes retournés chez les Kahn. [...]

[Le mercredi], nous avons rendu visite au grand rabbin. Il habite sur la «Place allemande», un chaos de maisons et d'écoles juives construites les unes sur les autres, habité par des Juifs ashkénazes de Hollande et d'Allemagne. Les maisons sont toujours construites les unes au-dessus des autres à Jérusalem et le toit de la maison d'en-dessous est aussi les fondations d'une autre. Le complexe où habite le rabbin est particulièrement propre et bien tenu. Il habite tout en haut sur une colline couverte de maisons et a la plus belle vue de la terre sur une terrasse qui entoure la maison et surplombe un abîme et des foules de gens. [...] Cet homme corpulent est un mizrahi ${ }^{19}$ et un opposant à tous les sionistes. Pour sa piété et son savoir, il est entretenu par des pieux du monde entier, il vit ici depuis des décennies des aumônes qui lui arrivent du monde entier et il vit apparemment très agréablement et bien. [...]

Aujourd'hui c'est le jour du facteur. [...] Demain, vendredi, je pense me rendre à Tel-Aviv. De là, j'irai ensuite dans les colonies. Pour huit jours. [...]

Votre vieil ours des mers, Theodorus Jerusalemitius.

\footnotetext{
${ }^{19}$ Les Juifs mizrahi sont les Juifs descendants des communautés juives du MoyenOrient. Les plus religieux étaient opposés à l'édification d'un État juif en Palestine qui ne pouvait être réalisée qu'aux temps messianiques. Ils vivaient uniquement d'étude et de prières, entretenus par la charité des communautés juives du monde entier. C'était le principal reproche que leur faisaient les pionniers sionistes qui, au contraire, s'installaient en Palestine pour travailler la terre, développer une économie juive et finalement instaurer un État juif.
} 
Theodor Lessing (1872-1933)

(site wikipedia.org)

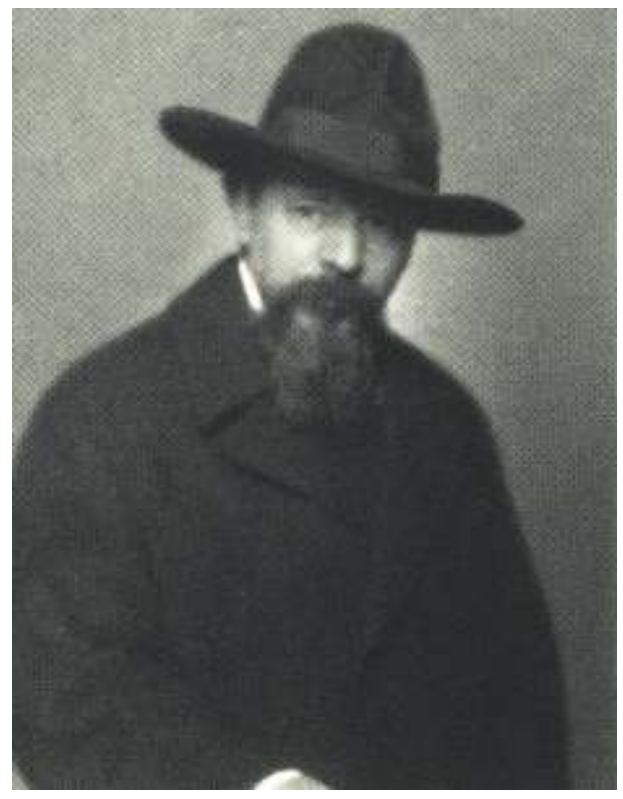

Dans les années 1920 